\title{
Object and Its Worry about Existence
}

\author{
Martin Vlcek \\ eFunctionality
}

\begin{abstract}
The presented work describes principles of functioning of the object as a philosophical category on the basis of principles discovered in different, concrete objects. At the beginning, a supposition is thus made that principles are common and describe the fundamental mode of the organization of matter. The regeneration of its elements is the basic goal of the control of an object. The control systems try to allow elements to optimize their state depending on circumstances and occasionally in advance. The input system is the first one from these systems. It controls the input of material for the regeneration and at the same time is the source of primary information concerning its state in the environment and its development in time. The system of the control of the distribution of the energy named TOT is another element of the control. The system named EM0 controls the process of the regeneration on the basis of information about the successfulness of elements involved into the regeneration. Finally, the system named EM1 controls the dynamics of the boundary of the object on the basis of information describing the tension on this boundary. The second goal of the control of an object, besides the regeneration, is to optimize the existence of the object as whole. New global criteria arise and are used throughout the process of the control for this sake.
\end{abstract}

Keywords: object, existence, control, satisfaction, tension

\section{Introduction}

\subsection{Analogy}

The basic properties of every object are derived from the functioning of its control systems. These systems function on different levels with the same logic, even if they are working with different elements. However, goals and means are the same-in Refs. [1] and [4].

The submitted work also arises from this logic. It uses findings from analogical systems proved by extended calculations—in Refs. [2] and [3] —and finds and applies them on an object in general.

\subsection{The Satisfaction and the Successfulness}

Every system works with two basic quantities describing the state of elements and the state of the whole system-new object. These quantities are defined on the basis of inputting or outputting interactions into an element.

The satisfaction is a function of the evenness and of an average value of inputting interactions. The successfulness is a function of a sum of outputting interactions.

The satisfaction describes the state when necessary inputs for the existence of an element are stably and reliably supplied. The successfulness describes the involvement of an element into the system, the interest for

Martin Vlcek, RNDr. et RNDr., Ing., eFunctionality, non-profit research group, Prague, Czech Republic; main research field: Cybernetics. Email: martin.vlcek@efunctionality.eu. 
its activity.

\section{The Control of an Object}

\subsection{The Central Axe-the Regeneration}

The basic function of an object, its central axe, is besides the control of the existence of the object as whole the regeneration of its elements. It consists in not only the solving of sequels of their activities or changes in the environment, but also in a preparation to them, occasionally in an avoidance of unwanted states.

Elements enter into a variety of interactions and structures. It is important that an element itself prefers some states and interactions over others; it means it is more stable in them. It is also important with what elements, of what quality it enters into interactions. All this determines the stability, satisfaction of an element. Moreover, a certain dynamics of states serves as a communicative means and it is possible to say that the world of interactions of elements is very variable and dynamic. This world is submitted to the process of the regeneration.

At the beginning of the system of regeneration stems the boundary of the object and on it the Input system as a supplier of material inputs. The Input system is also a source of basic impulses for the next control.

The process of regeneration is on its first level controlled through the distribution and production of the energy.

There are two other higher control systems around the central axe and around its energetic control, too. Both work with the successfulness of elements. The successfulness of individual elements in the regeneration evaluates the system named EM0. The satisfaction of the object as whole, stemming from the tension on the boundary, solves the system named EM1.

\section{Input System ENS}

The Input system represents a relatively autonomous and highly important part of the regulatory structure of an object, later on named ENS.

Besides the function connected with an input of material, sub-elements, it goes about the place of origin of impulses; it means of primarily information about the state of the regeneration, respectively of its suppositions-inputs to be at disposal of the object.

Impulses from ENS enter through the way of communicative substructures into the object and form a gradient in what work higher control systems.

There is a bifurcation of ways in ENS. The material part continues by a more or less controlled manner into positions for the subsequent processing. The signal part enters by the pathway of informative substructures and brings the information concerning the state of individual parts of ENS.

ENS in general is formed from two parts. Both are a source of sub-elements. The first part, later on named the inner boundary, forms sub-elements through its own sources. Its possibilities are limited by inner sources of the object and thus in the case of necessity the second part helps.

The second part of ENS takes sub-elements from the environment and later on is named as an outer boundary.

\section{TOT}

\subsection{The Control of the Axe-TOT}

The lowest level of the control of the axe is connected with the production and the distribution of the 
energy. Its particularity is among others in the fact that it doesn't stem from the feedback from individual elements executing the regeneration, but from the overall satisfaction of the object with the regeneration. This control system is later on named TOT.

The role of the TOT is to optimize the process of the regeneration from the energetic point view. It means to evaluate the presence of certain indicators of the state of the regeneration and to react in a manner to advance the overall regeneration. TOT reacts not only on the state of these indicators but on their dynamics, when a volatility of a state is just a demonstration of a certain status of the regeneration.

TOT in its activity interconnects the energetic system of the object and of elements. It mainly determines the distribution into the consumption and into the reserves, into object and into elements.

\section{EMO}

\subsection{Regeneration}

The task of the EM0 is to control structures executing the regeneration. It thus doesn't evaluate the successfulness of the regeneration itself, its final effect. This is made through the TOT, but on the basis of the successfulness of individual participating elements and their complexes optimizes the process of the regeneration itself.

An element mostly forms for its activity in the regeneration some complex with other elements. Thus, an involvement of an element into the regeneration is mostly measurable throughout a successfulness of some complex.

\subsection{Information}

In contrast with TOT, where a feedback happens on the basis of global indicators of the regeneration, the information coming from complexes of elements is the origin for the control in EM0. It is probable that this information is built upon some indicator of the consumption of the energy: It means about the involvement into the regeneration.

It is important that the relevant information is not only the concentration of these products in a concrete place but also their volatility at time. EM0 intervenes then into areas with not only high consumption of energy but preferably into area with a volatile one, by other words, into areas with a high and volatile demand, successfulness.

\subsection{Aggregation}

Information from ENS goes through EM0 and is aggregated. The result of this is an evaluation of the global orientation of the necessary reaction, of the process of the regeneration.

In general, it is not a mechanical solution of the actual state, but a finding out of an optimal strategy. This strategy starts to be gradually realized, through a way of a desegregation it is transcribed on a local level and using a feedback about the successfulness is occasionally modified. It can be a strategy, or step not mechanically following up from the situation, but through the "memory" of EM0, e.g., it precedes some unwanted state.

\subsection{Reconstruction}

It goes about two aspects of the control throughout the optimization of the process of the regeneration. In first, one the overall potential of the regeneration, e.g., the energy, is redistributed depending on the overall 
necessity of the object. It is necessary to find out the most exposed areas of the regeneration and to intervene into them. Inversely, it is possible to damp areas less active. Thus, it goes about a quantitative control.

In the second aspect, it goes about a qualitative control, when successful areas are strengthened because the success means the demand. New analogical elements or complexes are directed beside successful elements or complex of them and an analogical success is expected. Inversely is damped the directing of them into non-demanded areas.

By this way also the reconstruction of the structure of TOT arises. Its successful areas and ways of the control are strengthened and vice versa. The energetic control of the regeneration realized through TOT is thus interpreted as any other activity in the regeneration.

\section{EM1}

\subsection{The Task of EM1}

The task of EM1 is to control events on the boundary of the object.

A tension arising on the boundary in the case when two next positions supplying similar sub-elements for the regeneration receive non-similar reaction from this structure of the regeneration is an impulse for its activity. The basis of the activity of EM1 is to reconstruct the boundary in a manner to cancel this tension.

Similarly, it is possible to understand the activity of EM1 on the boundary as a leveling of the successfulness, at time and in place. Two similar positions should be similarly successful.

\subsection{Principle of the Functioning of EM1}

The general principle of the functioning of EM1 is as follows. A finding out of a maximal, resp. the most important tension on the inner boundary happens throughout the aggregation. The working boundary is used for it. This information is projected through the way of desegregation into the movement, what at this case means a redistribution of intensities of inputs on the outer boundary among individual parts and sub-elements. Thus, the tension on the inner boundary is solved through the reconstruction of the outer boundary.

It is important that EM1 in its work is controlled by global indicators reflecting the state of the distribution of tension on the primary boundary. It goes about the global tension and satisfaction.

\subsection{The Satisfaction}

The satisfaction of an object, its main indicator, is a function of the evenness of the inner boundary, it means of the level and volatility at time of the successfulness of its positions.

The inner boundary thus thanks to its distribution of the successfulness and its dynamics is a criterion and at the same time the hint how to proceed on. EM1 evaluates on the basis of the distribution of the successfulness the real cause of the problem, of a certain dissatisfaction and chooses the strategy depending on it.

This is the intervention of EM1 in the outer boundary, which provokes changes in intensities of inputs what in their turn have as a sequel a solution of the dissatisfaction of the object. Then this controlled change of intensity of interactions is another side of the overall movement of the object in its material environment.

Throughout its work, EM1 has to make provision for the tension on the outer boundary: It means to do the movement in a manner that a too high tension doesn't arise, or the tension would be only temporally at the time of the cancelling of the inner tension. 
The intervention on the outer boundary itself is similar as in the case of EM0, thus, it is a controlled intervention of the fund depending on the distribution of the successfulness.

\subsection{The Working Boundary}

The work of EM1 consists in a usage of an internal boundary forming a transfer among the inner and outer boundary.

The working boundary ensures that EM1 realizes in a given moment only one global movement and thus a mutual interference doesn't happen. Similarly only one the most important criterion is solved at a given moment. This is reached through the fact that the inputting tension, which means a non-corresponding successfulness at similar inputs, is aggregated to higher levels and only one global, summarily describing tension is found. Only this tension enters into the "consciousness" and is processed. It means that a movement, a reconstruction of the outer boundary, is found out what will cancel this tension.

It is possible to imagine that local tensions or unevenness of successfulness spread from different informative sources into the working boundary. These partly information are connected, partly on the basis of the last experience and a complex tension arises. If it is sufficiently strong, it will arrive till the working boundary. The process of the aggregation is not only an interconnection but also a suppression of concurring tensions being not a part of a successively identified global tension.

When the tension enters into the consciousness, it "turns" its course and a process of desegregation starts into a movement. A strengthening of well-tried strategies of identification of the tension, which means of procedures used throughout the way "up," is also a part of this maneuver. If throughout a gradual realization of the movement an abolishment of individual tensions will arise, strategies identifying this tension throughout the way up will be strengthened. There is thus used a feedback when the success of the identification of a tension is verified throughout its abolishment.

\section{The Holistic Principle}

The boundary of an object is a place where a new object, new quality arises. A new quality arises through the fact that a notion of the consciousness happens; it means the only one aggregated and evaluated tension what is solved and what thus influences the next behavior of the object. There is no more a simple sum of individualities more or less cooperating, but only one common strategy. And this consciousness has not to solve only the actual representation of the highest tension but perhaps of something been only in suspicion.

Besides the global satisfaction, what determines a gradient for the movement of the object, the global tension-the consciousness, gives an impulse allowing to orienting itself throughout this movement, to find solutions.

The third global quantity is the result of the aggregation in EM0. It shows the global success of the regeneration evaluated on the basis of the information coming from individual elements or complexes of them. Only this quantity allows to exactly control the process of the regeneration and to fulfill the first goal of the object-the care for elements.

The behavior of the object is controlled throughout a mutual cooperation of these three quantities. Quantities connected with EM1 are dominant and the quantity connected with EM0 serves to the choice of strategies from the point of view of the energetic demand. 


\subsection{The Speech}

The object can enter into communication with other objects thanks to the activity on the outer boundary - mainly with objects of the same kind. The activity of the boundary is not in this case oriented to the solving of a tension of the inner boundary, but for the communication of global states of the object. Thus, it goes mainly about the communication of the global satisfaction and all of its nuances, and occasionally also of the global tension; it means of the content of the consciousness.

The substance of the communication consists in the fact that the second object learns to follow some otherwise unimportant dynamic of the outer boundary and connect it with a state of the first object. Then it can be used for other interactions, concretely for a modification of supplying inputs, which better responds to the interest of the consumer, etc.

Thus, the speech is another mechanism which can function only in a holistic environment. It is a communication only among objects as whole, not among their elements.

By the way, the man can use this speech in the process of cognition of any object. After understanding of the language, he can monitor by a principal way the object and nota bene to influence it, too.

\section{New Quantities}

Any quantity used by the object, e.g., physical one, has to have its beginning in the structure and functioning of the object. The object can understand only this kind of quantity.

And the understanding is given by the fact that this quantity has a meaning either for the structure of the regeneration or for the object as whole, means for events on its boundary.

\subsection{The Time and the Space}

E.g., time is somehow given by the activity of TOT. The more energy is oriented into the regeneration, the more quickly it runs and thus the more runs its time. Of course, there are different ways of regenerations differently exigent for the energy, but TOT doesn't solve the structure of the regeneration and in addition; the time as other quantity in the object exists only in its approximate form. E.g., the physical time is only a generalization of a real time.

Similarly it is with the distance. It is perhaps connected with the activity of EM1 and reflects events on the outer boundary. Only there arises a quantity defining the distance as a function of the similarity and of intensities of inputs, of their successfulness. The sum of interactions with the environment is then simplified or transformed into the physical notion of a distance. The reason for it is perhaps operational, when for the functioning of EM1 is convenient to describe the overall relation to some object in the environment. But of course the distance as something absolute doesn't exist, similarly as time. It is only an operational instrument of the object.

There is introduced the notion of the power on the outer boundary, as a reflection of the tension between elements or areas. For new, it is a certain operational simplification of the description of a relation of two areas or elements.

And finally the energy is the measure of the activity of EM0 throughout its quantitative control.

\section{The Process of an Arising of a New Object}

It seems that the object arises not only by a grouping of elements into the object, but at the same time 
inversely — by the influence of the object on involved elements. Elements in the object cease to be the original ones, their properties change.

And at the same time, it is probable that as a result of this, another object ceases to exist on a different level, because these new properties are already not compatible with its functioning. Its control is already not interested for elements.

Thus, it is possible to imagine that there is a constant dynamics of an overflow of organizations to different levels and to different objects, when a rise of one is connected with extinction of others.

\section{Conclusion}

The submitted paper reflects functioning and observing of an object as whole on the basis of analogies. It is a way stemming from the fact that an object exists as an integral object being connected by common interests of building parts and by solving them.

From the point of view of a human, these activities are non-observable, but they are very important for the existence of an object. The problem with the observation of these small relevancies is in the fact that it is necessary to have to the disposal a great deal of receptors but mainly and it is necessary to use new methods of processing.

Nevertheless, the cognition of an object as whole, of a new entity, is quite fundamental. Besides the fact that it allows to understand new structures and mainly new logics of their functioning, it will allow to enter into a new level of communication with the observed object and to begin to understand it also by a new way- to let it "narrate about itself."

\section{Works Cited}

Vlcek, Martin. "Functioning of the Object.” Unpublished material, 2008.

---. "Movers of the Economy-Model of Interactions.” Developments in Economic Theory and Policy Proceedings. Bilbao, Spain, 2010.

---. "New Object Discovered through the Stock Market.” International Conference on Economics and Finance Research Proceedings. Seoul, South Korea, 2014.

---. “Firm without Subordinations.” American Journal of Economics 5.3 (2015). 